\title{
Correction to: Basic Theory and Laboratory Experiments in Measurement and Instrumentation
}

\section{Correction to:}

\author{
A. Cataldo et al., Basic Theory and Laboratory \\ Experiments in Measurement and Instrumentation, \\ Lecture Notes in Electrical Engineering 663, \\ https://doi.org/10.1007/978-3-030-46740-1
}

The original version of the book was inadvertently published with an incorrect spelling of the first name of the author Alessandro De Monte. The first name was corrected from Alessando to Alessandro. The corrected book has been updated with the changes. 